EXTRACTION OF POWER LINES AND PYLONS FROM LIDAR POINT CLOUDS USING A GCN-BASED METHOD
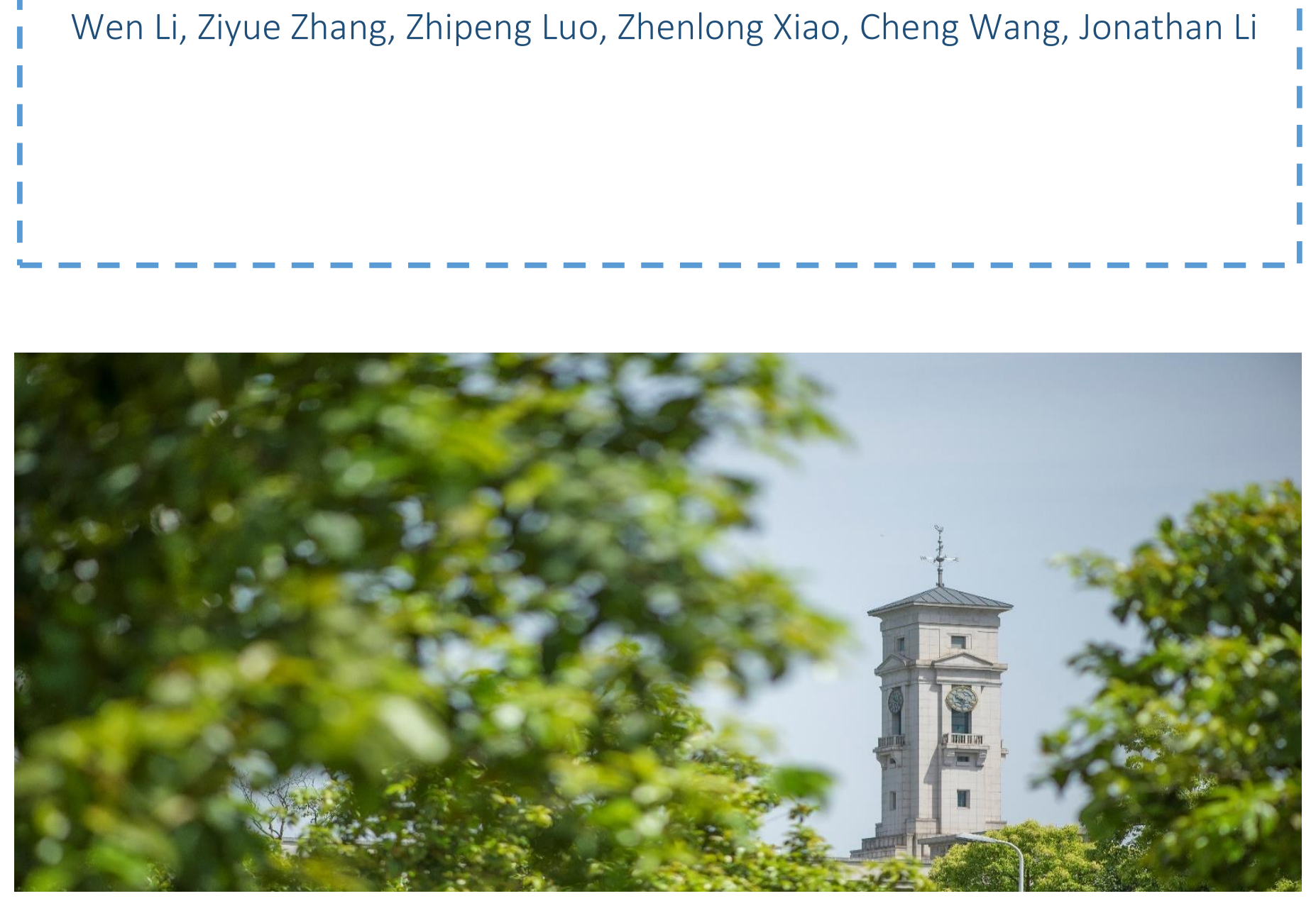
University of Nottingham Ningbo China, 199 Taikang East Road, Ningbo, 315100, Zhejiang, China.

First published 2020

This work is made available under the terms of the Creative Commons Attribution 4.0 International License:

http://creativecommons.org/licenses/by/4.0

The work is licenced to the University of Nottingham Ningbo China under the Global University Publication Licence:

https://www.nottingham.edu.cn/en/library/documents/researchsupport/global-university-publications-licence-2.0.pdf 


\title{
EXTRACTION OF POWER LINES AND PYLONS FROM LIDAR POINT CLOUDS USING A GCN-BASED METHOD
}

\author{
Wen Li $^{1}$, Ziyue Zhang ${ }^{3}$, Zhipeng Luo ${ }^{1}$, Zhenlong Xiao ${ }^{1}$, Cheng Wang ${ }^{1}$, Jonathan Li $^{1,2 *}$ \\ ${ }^{1}$ Fujian Key Laboratory of Sensing and Computing for Smart Cities, School of Informatics \\ Xiamen University, Xiamen, FJ 361005, China \\ ${ }^{2}$ Department of Geography and Environmental Management and Department of Systems Design \\ Engineering, University of Waterloo, Waterloo, Ontario N2L 3G1, Canada \\ ${ }^{3}$ School of Computer Science, University of Nottingham Ningbo China, Ningbo, ZJ 315100, China \\ *Corresponding author: junli@xmu.edu.cn and junli@uwaterloo.ca (J. Li)
}

\begin{abstract}
The routine power line inspection is critical to maintain the reliability, availability, and sustainability of electricity supply. As a key part of inspection, power lines and pylons extraction is essential for resource management and power corridor safety, especially in the mountain regions. In this paper, we proposed a deep learning based method to extract power lines and pylons using ALS point clouds. First, a structure information preserved module is designed to mine the relationship of local neighborhood points. Then, a graph convolutional network (GCN) is used as basic module to extract point features. Finally, three categories, power lines, pylons and other objects are segmented from input point clouds. In addition, we provide an effective data enhancement strategy to generate enough samples to train the proposed model. Experiments demonstrate that our method achieves competitive results in accuracy and efficiency. The overall accuracy and mean time are $99.1 \%$ and 9.3 seconds.
\end{abstract}

Index Terms - Power line, pylon extraction, ALS, point cloud, graph convolutional network

\section{INTRODUCTION}

Nowadays, electricity plays an indispensable role in social production, thus it is necessary to arrange power line patrol regularly to ensure the operation safety. Because ALS system can acquire 3D shape information accurately and efficiently in a large area, ALS point clouds has become a high priority data for many related tasks, such as power line classification, extraction and reconstruction $[1,2]$. As a key part of these tasks, power lines and pylons extraction from ALS point clouds can be achieved by two ways, the manual-designedfeature based method [3, 4] and property-based method [5, 6]. However, point features extracted by these two techniques are all shallow and low-discriminative, which would be of great negative impact on the power lines extraction. Recently, with the increase of available data and the improvement of

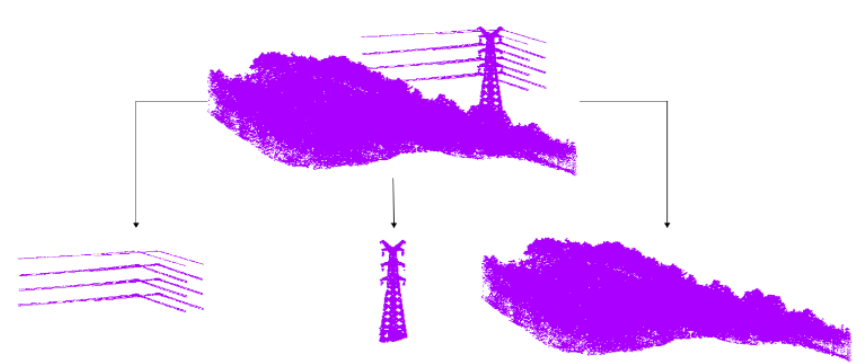

Fig. 1: Power line and pylons extraction from ALS point clouds (top) with segmented powerlines (bottom left), pylon (bottom middle), and other objects (bottom right).

hardware performance, deep learning (DL) [7] shows its powerful feature description ability in wide applications, especially in computer vison, such as the image and 3D point cloud processing $[8,9]$. Therefore, in this paper, we aim to investigate the application of DL in power lines and pylons extraction using ALS point clouds.

The key issue of applying DL in 3D data lies in the design of appropriate representation. Existing representations can be divided into three categories, voxel based, view based and point based methods [10]. These methods have achieved promising results in some synthetic datasets, such as ModelNet [11]. However, they may not be appropriate in processing unordered ALS point clouds. In this work, considering that the local geometric information is preserved in the relationship of neighbor points, we utilized the graph data structure to present the ALS point clouds and developed a new approach based on the graph convolutional network (GCN) to extract the power lines and pylons. Especially, we firstly designed a structure information preserved module to mine the relationship of local neighborhood points. Then, we used the graph convolutional network (GCN) as basic module to extract deep point features. Finally, three categories, power lines, pylons and other objects are segmented from input point clouds. In addition, we provide an effective data enhancement strategy to generate enough samples to train the proposed model. Fig. 1 shows the application of our approach 


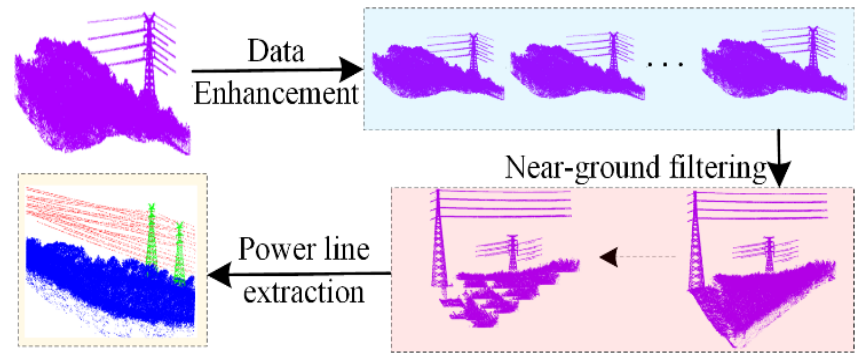

Fig. 2. The flowchart of our method. It consists of three parts: data enhancement, near-ground filtering and feature extraction.

in extracting power lines and pylons.

The rest of this paper organized as follows. Section 2 presents the related works. Section 3 provides the details of the proposed method. Section 4 shows and discusses the experimental results. Section 5 concludes the paper.

\section{RELATED WORK}

Existing power line extraction methods can be divided into two categories: mathematical estimation and classificationbased methods. In the first kind of methods, Hough transform and random sample consensus (RANSAC) were usually used to extract power lines points. Guan et al. [6] proposed a method to determine the non-ground points based on incident angle and elevation, then combined with density and shapeand-size filter to extract power lines, but results are affected by data density. Yadav et al. [12] used 2D point density-based refinement to remove building and tree points, and then used Hough transform to extract power lines. However, the result is sensitive to parameter settings. Lehtomaki et al. [13] voxelied the data then used principal components analysis (PCA) and RANSRC to extract power lines and pylon points, but voxelization loses some information. Classificationbased methods usually use machine learning methods, such as support vector machine (SVM) and random forest (RF). Wang et al. [14] constructed power line corridors through filtering and RANSAC. Then selected slant cylindrical neighborhood to extract geometric features. Finally, it used SVM to obtain results. However, this algorithm does not consider ground fluctuations. The Joint-Boost classification and 26 features were used in [15] to classify point clouds, but it need to use image segmentation results to improve accuracy. Peng et al. [16] built feature vectors based on density, elevation and vertical contour features. Then it used RF to obtain classification results. However, results generated by this method were sensitive to radius of feature extraction.

In summary, features extracted by the above methods are shallow and low-discriminative. On the other hand, applications of DL in 3D point cloud [9, 17] has shown its power in learning deep features from 3D data. Therefore, in this work, we seek the possibility of applying DL in ALS point clouds using the graph data structure. To the best of our knowledge, this is the first application of GCN in power lines and pylons extraction.

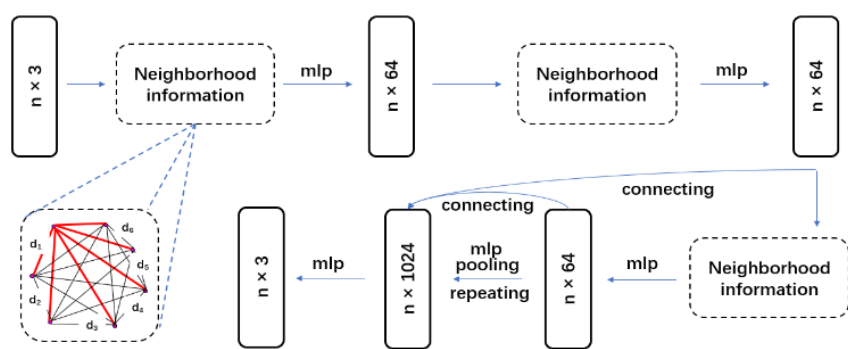

Fig. 3. The network of GCN used in our method. The graph inside the dotted line in the lower left corner represents the neighborhood information extraction module, and the red line represents the distance from the sample point to each point.

\section{METHOD}

As shown in Fig. 2, our method consists of three parts: data enhancement, near-ground filtering and feature extraction.

\subsection{Data enhancement}

Supervised approaches usually require a lot of training data. However, large number of samples are often unavailable in real life. In our work, to solve the problem of insufficient data, we develop a data enhancement strategy. Specifically, we randomly cut ground with a length and width of 100 meters from the original data, and generated a total of 10 scenes. Then, we selected pylons with obvious structures and maintained their power line connections. They were randomly placed on the ground and ensured that there were 2 to 3 pylons in each scene, which greatly increased the number of samples. We generate a total of 150 training samples and randomly selected 30 samples for verification. The test samples are real scenarios.

\subsection{Near-ground filtering}

The imbalance between target and background is also an issue in object detection. The focal loss may be an available idea in solving this problem. However, after we tried this idea, the result was still unsatisfactory. Therefore, we sought a near-ground filtering method to quickly reduce ground points, while it can preserve the pylon and power line points.

Specifically, we found that pylons and power lines are located above ground, but it is hard to directly use a single threshold to reduce ground points. Therefore, we developed a multi-threshold strategy. Firstly, the input is segmented into square parts with side length of 20 meters. Then, the points in each square part are sorted in descending order according to the $z$ axis. Finally, the first $n_{\text {new }}$ points are taken in the following way:

$$
n_{\text {new }}=\left\{\begin{array}{c}
15000, \text { if } n \geq 20000 \\
n-5000, \text { if } 12000 \leq n<20000 \\
n, \text { if } n<12000
\end{array}\right.
$$

where $n$ is the original number of points in each part. The principle of this strategy is that the ground points are dense 
Table 1. Key statistics of test scenarios

\begin{tabular}{c|c|c|c|c}
\hline \multirow{2}{*}{ Statistics } & \multicolumn{3}{|c|}{ Elevation $(\mathrm{m})$} & \multirow{2}{*}{$\begin{array}{c}\text { Density } \\
\text { (points/m² }\end{array}$} \\
\cline { 2 - 4 } & Maximum & Minimum & Mean & 42 \\
\hline Area_1 & 142.27 & 54.95 & 95.99 & 70 \\
\hline Area_2 & 187.67 & 58.17 & 107.91 & 48 \\
\hline Area_3 & 152.58 & 57.11 & 94.46 & 39 \\
\hline Area_4 & 184.16 & 69.47 & 106.98 & \\
\hline
\end{tabular}

and other objects are distributed in areas with relatively high elevations.

\subsection{GCN network structure}

Local feature plays important rale in 3D point cloud labeling. Although the method proposed in [17] can handle point cloud data well, the performance is still far from satisfy and many improved versions have been proposed. In our method, we developed a GCN-based network to extract the local features efficiency. More specifically, as shown in Fig. 3, the input with shape of $n \times 3$ is firstly put into the neighborhood information extraction module, where $\mathrm{n}$ is the number of points and 3 denotes the coordinates. Then, a multilayer perceptron (MLP) is used to extract the point features, following by two blocks with neighborhood information extraction and MLP modules. Besides, the skip connection is used to preserve more details. Finally, the global features are extracted through a max-pooling operation.

\section{RESULTS AND DISCUSSION}

\subsection{Dataset description and implementation}

Our data was collected by HawkScan X3, with fight height of $150 \mathrm{~m}$, speed of $24 \mathrm{~m} / \mathrm{s}$ and scanning frequency of $400 \mathrm{kHz}$. We chose four real scenarios as test data. For a more intuitive understanding of the data, several important statistics were listed in Table 1. It can be inferred from the elevation mean and difference that those scenarios are undulating on the ground. Specifically, the maximum elevation difference exceeds $120 \mathrm{~m}$. What's is worse, it can be seen from the data density that data is sparse. For extraction methods that are sensitive to data density and threshold settings, these are challenging.

We implemented experiments in Ubuntu 16.04, Inter(R) Core (TM) i7-7700K 4.20GHz, Tesla P100-PCIE-16GB and $16 \mathrm{~GB}$ memory. We calculated the overall accuracy $(\mathrm{OA})$ and the running time (RT). Besides, F1-score were also used to evaluate the extraction performance. We designed two comparative experiments, which use PCA to calculate dimensional feature and use feature with small angles between power lines points to extract power lines and pylons points, called Baseline_1 and Baseline_2, respectively. These two baselines used CSF [18] to separate ground and nonground points, then extracted the power lines and pylons points by setting reasonable thresholds. Those two methods were used as comparison because they are representative in traditional algorithms.
Table 2. Comparison of raw point cloud data and filtered results

\begin{tabular}{c|c|c|c|c|c|c}
\multirow{2}{*}{ Num } & \multicolumn{3}{|c|}{ Raw point clouds } & \multicolumn{3}{c}{ Filtered results } \\
\cline { 2 - 7 } Area_ & Line & Pylon & Other & Line & Pylon & Other \\
\hline Area_1 & 4,934 & 5,705 & 411,485 & 4,927 & 5,705 & 314,249 \\
\hline Area_2 & 11,861 & 18,186 & 672,207 & 11,856 & 17,764 & 345,380 \\
\hline Area_3 & 3,632 & - & 477,330 & 3,623 & - & 345,380 \\
\hline Area_4 & 5,765 & - & 389,458 & 5,761 & - & 318,779 \\
\hline
\end{tabular}

Table 3. Comparison of overall accuracy (OA) and running time (RT)

\begin{tabular}{c|c|c|c}
\hline OA/RT & & \\
Area & Ours & Baseline_1 & Baseline_2 \\
\hline Area_1 & $\mathbf{9 8 . 9 / 9 . 8}$ & $97.3 / 29.6$ & $98.2 / 46.5$ \\
\hline Area_2 & $\mathbf{9 7 . 6 / 8 . 3}$ & $90.6 / 30.7$ & $94.5 / 61.1$ \\
\hline Area_3 & $\mathbf{9 9 . 8 / 1 0 . 3}$ & $99.7 / 28.7$ & $99.8 / 31.8$ \\
\hline Area_4 & $\mathbf{9 9 . 9 / 8 . 8}$ & $96.8 / 25.2$ & $97.6 / 46.1$ \\
\hline Average & $\mathbf{9 9 . 1 / 9 . 3}$ & $96.1 / 28.6$ & $97.5 / 46.4$ \\
\hline
\end{tabular}

Table 4. Quantitative results in F1-score (\%)

\begin{tabular}{c|c|c|c|c|c|c}
\hline \multirow{2}{*}{ F1 (\%) } & \multicolumn{2}{|c|}{ Ours } & \multicolumn{2}{c|}{ Basline_1 } & \multicolumn{2}{c}{ Basline_2 } \\
\cline { 2 - 7 } & Pylon & Line & Pylon & Line & Pylon & Line \\
\hline Area_1 & $\mathbf{7 4 . 2}$ & 82.8 & 44.3 & 64.8 & 60.1 & $\mathbf{8 9 . 8}$ \\
\hline Area_2 & $\mathbf{7 0 . 0}$ & $\mathbf{8 3 . 5}$ & 11.7 & 16.1 & 29.2 & 65.8 \\
\hline Area_3 & - & 94.1 & - & 89.5 & - & $\mathbf{9 5 . 2}$ \\
\hline Area_4 & - & $\mathbf{9 6 . 2}$ & - & 58.5 & - & 67.4 \\
\hline
\end{tabular}

\subsection{Experiment results}

Table 2 provides the comparison results of the number of points in raw and filtered point clouds. The results demonstrate that our filtering method can reduce part of the other clutter points, while retain most of the power lines and pylons points.

As shown in Table 3, our method outperforms the comparison methods in terms of OA and RT. Specifically, our method achieves the best OA in every area. Furthermore, compared with Baseline_1, our method obtains an improvement of 3\%. In addition, the RT of our proposed method was less than other two baselines. The average running time of our method was 9.3 seconds, which was onethird of that of Baseline_1 and one-fifth of that of Baseline_2.

Table 4 shows F1-score results generated by our method and baselines. Obviously, our method obtains excellent F1 values. More specifically, on Area_1, the F1-score of pylon extracted by our method was $74.2 \%$, which was nearly $30 \%$ higher than that of Baseline_1. On Area_2, our method achieved the best performance in terms of pylon and line extraction. On Area_3 and Area_4, our method also obtained competitive results. In addition, we visualized several experimental results. As shown in Fig. 5, our method was able to extract targets pretty well, especially the pylon points in both relatively simple and complex scenes. Meanwhile, the height and structure information of the pylon were well preserved. The reason for all these promising results of our method lies in the consideration of the relationship of neighborhood points. The local features learnt by GCN in our method enhance the ability of preserving the structural characteristics of the pylons and power lines. 

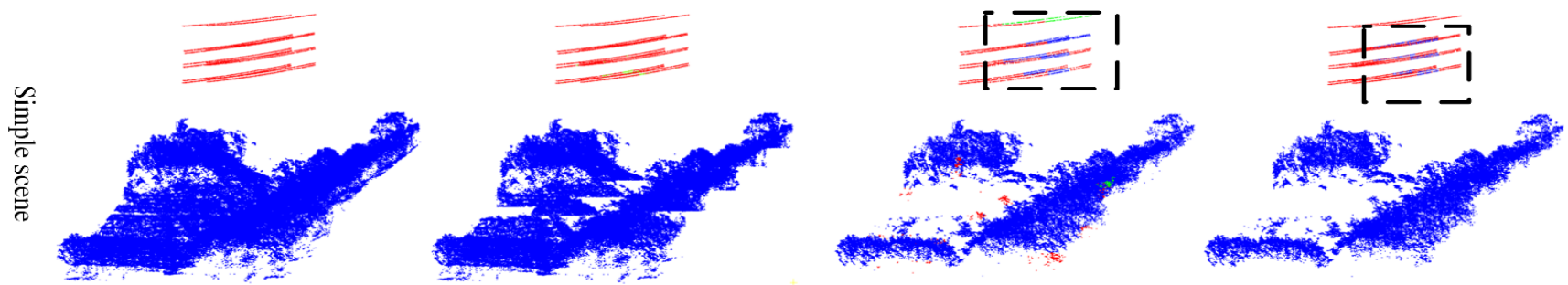

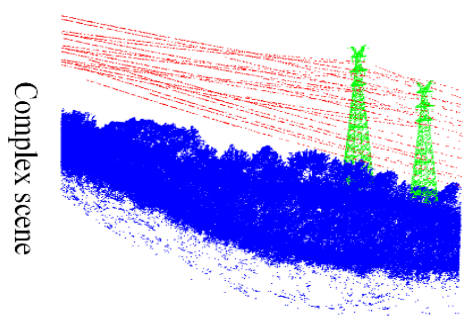

(a) Ground truth

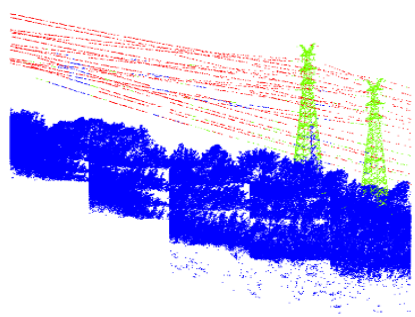

(b) Our method

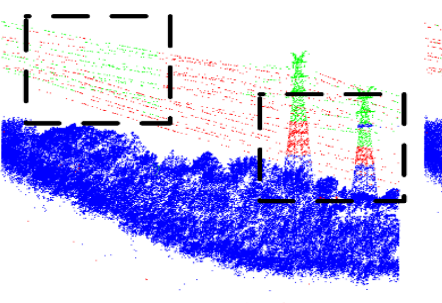

(c) Baseline_1

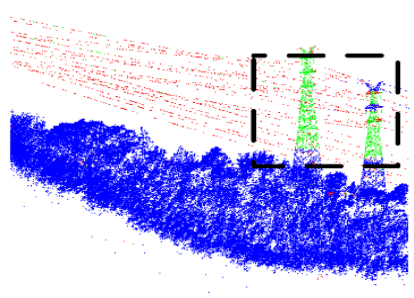

(d) Baseline_2

Fig. 5. Extraction results of (a) Ground truth, (b) Our method (after near-ground filtering), (c) Bseline_1 (after CSF [18] filtering), (d) Bseline_2 (after near-ground filtering). Our method can well extract targets, especially the pylon points, on both relatively simple and complex scenes. The areas marked by the black boxes are areas with more mislabeling error point.

\section{CONCLUDING REMARKS}

In this paper, we presented a new approach using a GCNbased network to extract power lines and pylons points. We solved the problem of insufficient samples and imbalance by data enhancement strategy and near-ground filtering, respectively. We achieved excellent results in accuracy and efficiency. What's more, due to the consideration of neighborhood information, we preserved the structure information of the extracted pylons. In some complex scenarios, some power lines points were mistakenly extracted as other points. In the future work, we will try to solve this problem by add dimensional feature.

\section{ACKNOWLEDGEMENTS}

This work was supported in part by the National Natural Science Foundation of China under Grant 41871380.

\section{REFERENCES}

[1] S. Xu and R.S Wang, "Power Line Extraction From Mobile LiDAR Point Clouds," IEEE J. Sel. Top. Appl. Earth Observ. Remote Sens., vol. 12, no. 2, pp. 258-265, 2017.

[2] L. Matikainen, L. Matti, E. Juha, et al., "Remote sensing methods for power line corridor surveys," ISPRS-J. Photogramm. Remote Sens., vol. 119, pp. 10-31, 2016.

[3] B. Guo, Q. Q Li, X. F Huang, et al., "An Improved Method for Power-Line Reconstruction from Point Cloud Data," Remote Sens., vol. 8, no. 1, pp. 36-53, 2016.

[4] H.B Kim and G. Sohn, "Point-based classification of power line corridor scene using random forests," Photogramm Eng. Remote Sens., vol 79, no. 9, pp. 821-833, 2013.

[5] L. L Zhu and H. Juha, "Fully-Automated Power Line Extraction from Airborne Laser Scanning Point Clouds in Forest Areas," Remote Sens., vol. 6, no. 11, pp. 11267-11282, 2014.
[6] H.Y Guan, Y.Y Yu, J. Li, et al., "Extraction of powertransmission lines from vehicle-borne lidar data," Remote Sens., vol. 37, no. 1, pp. 229-247, 2016.

[7] Y. Lecun, Y. Bengio and G. Hinton, "Deep learning," Nature, vol. 521, no. 7553, pp. 436-444, 2015.

[8] A. Krizhevsky, I. Sutskever and G. Hinton, "Imagenet classification with deep convolutional neural networks," Commun. ACM, vol. 60, no. 6, pp. 84-90, 2017.

[9] Y. Guo, H. Wang, Q. Hu, H. Liu, L. Liu, M. Bennamoun, "Deep learning for 3D point clouds: A survey," arXiv:1912.12033 [cs.CV].

[10] Z. Luo, J. Li, Z. Xiao, et al. "Learning high-level features by fusing multi-view representation of MLS point clouds for 3D object recognition in road environments," ISPRS-J. Photogramm. Remote Sens., vol. 150, pp. 44-58, 2019.

[11] Z. Wu, S. Song, A. Khosla, et al. "3D ShapeNets: A deep representation for volumetric shapes," in Proc. IEEE CVPR, 2015, pp. 1912-1920.

[12] M. Yadav, C. G Chousalker, "Extraction of power lines using mobile LiDAR data of roadway environment," Remote Sens. Appl.: Soc. Environ., vol. 8, pp. 258-265, 2017.

[13] M. Lehtomaki, A. Kukko, L. Matikainen, et.al., "Power line mapping technique using all-terrain mobile laser scanning," Autom. Constr., vol 105, pp. 1-16, 2019.

[14] Y.J Wang, C. Qi, L. Liu, et.al, "Supervised Classification of Power Lines from Airborne LiDAR Data in Urban Areas," Remote Sens., vol. 9, no. 8, pp. 71-87, 2017.

[15] B. Guo, X. Huang, F. Zhang, et al., "Classification of airborne laser scanning data using JointBoost," ISPRS-J. Photogramm. Remote Sens., vol. 100, pp. 71-83, 2015.

[16] S. W Peng, X. H Xi, C. Wang, et.al, "Systematic comparison of power corridor classification methods from ALS point clouds," Remote Sens., vol. 11, no. 17, pp. 1961-1988, 2019.

[17] Y. Wang, Y.B Sun and Z.W Liu, "Dynamic graph CNN for learning on point cloud," ACM Trans. Graph, vol. 38, no. 5, pp. 1-12, 2019.

[18] W. M. Zhang, J. B. Qi, P. Wang, et.al, "An easy-to-use airborne LiDAR data filtering method based on cloth simulation," Remote Sens., vol. 8, no. 6, pp. 501-522, 2016. 